\title{
Mechanisms of vancomycin resistance in Staphylococcus aureus
}

\author{
Susana Gardete and Alexander Tomasz
}

Laboratory of Microbiology and Infectious Diseases, The Rockefeller University, New York, New York, USA.

\begin{abstract}
Vancomycin is a glycopeptide antibiotic used for the treatment of Gram-positive bacterial infections. Traditionally, it has been used as a drug of last resort; however, clinical isolates of methicillin-resistant Staphylococcus aureus (MRSA) strains with decreased susceptibility to vancomycin (vancomycin intermediate-resistant $S$. aureus [VISA]) and more recently with high-level vancomycin resistance (vancomycin-resistant $S$. aureus [VRSA]) have been described in the clinical literature. The rare VRSA strains carry transposon Tn1546, acquired from vancomycin-resistant Enterococcus faecalis, which is known to alter cell wall structure and metabolism, but the resistance mechanisms in VISA isolates are less well defined. Herein, we review selected mechanistic aspects of resistance in VISA and summarize biochemical studies on cell wall synthesis in a VRSA strain. Finally, we recapitulate a model that integrates common mechanistic features of VRSA and VISA strains and is consistent with the mode of action of vancomycin.
\end{abstract}

\section{Introduction}

Reduced vancomycin susceptibility and resistance in methicillin-resistant Staphylococcus aureus. By the end of the 1990s the relatively few multidrug-resistant and highly epidemic clones of methicillinresistant Staphylococcus aureus (MRSA) had become the most frequent causative agents of $S$. aureus disease in both hospitals and communities (1). In spite of the availability of several structurally different antibacterial agents, the therapy most frequently used for treatment of MRSA infections has remained the glycopeptide antibiotics, primarily vancomycin (2). From 1980 on, there was an abrupt and continued increase in the use of vancomycin in the United States and several countries (3), which seems to parallel the increasing frequency of MRSA infections in hospitals. This illustrates the enormous selective pressure highly focused on MRSA strains worldwide.

An MRSA isolate with decreased susceptibility to vancomycin was first reported in Japan in 1997 (4). The isolate had only a modestly increased minimum inhibitory concentration (MIC) value for vancomycin, in the range of $3-8 \mu \mathrm{g} / \mathrm{ml}$, and became known as vancomycin intermediate-resistant $S$. aureus (VISA). VISA isolates do not carry imported foreign genetic elements; rather, the increased vancomycin MIC values are related to mutations that appear in the invading pathogen during vancomycin therapy in vivo. VISA began to be reported with increasing frequency among MRSA isolates identified all over the world (for review, see ref. 5). In spite of their moderate increase in MIC value, vancomycin treatment of infections by VISA isolates often ended in treatment failure $(6,7)$. In 2002 , the first vancomycin-resistant $S$. aureus (VRSA) strain (with a vancomycin MIC value greater than $100 \mu \mathrm{g} / \mathrm{ml}$ ) was reported in the United States (8). Clearly the use of vancomycin therapy against such isolates would be impossible.

Comprehensive reviews of various microbiological and epidemiological aspects of VISA-type resistance have been published (5, 6, 9-12). Herein, we briefly discuss some of the mechanistic aspects of VISA-type antibiotic resistance, beginning with a case description that puts the emergence of a VISA mutant into an evolution-

Conflict of interest: The authors have declared that no conflict of interest exists. Citation for this article: J Clin Invest. 2014;124(7):2836-2840. doi:10.1172/JCI68834. ary context. Additionally, we summarize results of experiments that have begun to explore the biochemical mechanism of highlevel vancomycin resistance in $S$. aureus.

VISA strains: evolution and population structure. In the overwhelming majority of cases, VISA strains have emerged in patients with MRSA infections undergoing prolonged vancomycin therapy, which often ended in treatment failure (for review, see refs. 5-7). The emergence of one of the first VISA derivatives in the United States was documented in detail in the clinical and microbiological history of a 79-year-old patient with end-stage renal disease who received repeated courses of vancomycin therapy against an MRSA blood stream infection (13). The first MRSA isolate, named PC1 and recovered December 13, 1997, had a vancomycin MIC of $2.0 \mu \mathrm{g} / \mathrm{ml}$. However, examination of the PC1 culture by population analysis showed that it was heterogeneous; it contained subpopulations of bacteria with a frequency of $5 \times 10^{-4}$ that could grow on $2 \mu \mathrm{g} / \mathrm{ml}$ vancomycin. In addition, bacteria that could grow on $4 \mu \mathrm{g} / \mathrm{ml}$ vancomycin were also present, with a frequency of $6 \times 10^{-6}$. Upon readmission of the patient to the hospital on January 10, 1998, an MRSA isolate named PC2 was recovered from the bloodstream. The vancomycin MIC of PC2 was still 2 $\mu \mathrm{g} / \mathrm{ml}$, and the culture was still heterogeneous, but the size of the subpopulation capable of growing in the presence of $2 \mu \mathrm{g} / \mathrm{ml}$ had increased to $10^{-3}$. Approximately 2 months later, the same patient was readmitted to the hospital with a fatal MRSA blood stream infection. The vancomycin MIC of the strain named PC3 had now increased to $8 \mu \mathrm{g} / \mathrm{ml}$ and had become homogeneous: its MIC was shared by the entire bacterial population. Strains PC1 through PC3 shared an identical pulsed-field gel electrophoresis (PFGE) profile, indicating that these isolates were genetically identical. Like that of all other VISA isolates described in the clinical literature, the evolutionary history of PC1 through PC 3 begins and ends in a particular patient.

Increased vancomycin MIC and problems in chemotherapy. At first sight, the high frequency of failed vancomycin therapy (14-17) is surprising given the rather modest increase in the MIC value of the isolates and the fact that VISA strains showed decreased virulence potential when tested in a variety of animal models (18-21). Thus, a major contributor to the frequent lack of success of prolonged chemotherapy may be the poor health status of the patients, who 
are often elderly and have serious underlying health conditions $(2,5,18)$. Nevertheless, some data in the literature raise the possibility that factors in the invading bacterium may also contribute. Examination of the vancomycin MIC value of MRSA strains that were recovered from patients with failed therapy showed that therapeutic failure correlated significantly with the vancomycin MIC value of the strains, even when this antibiotic was not used for therapy $(15,16,22-24)$. These data suggest that the vancomycin susceptibility profile of MRSA strains correlates with some asyet-unidentified aspects of the invasive potential of the bacteria.

Population structure of VISA isolates. A characterization of VISA isolates using the method of population analysis first developed for the characterization of MRSA strains with heterogeneous resistance to $\beta$-lactam antibiotics (25) has led to the identification of "hetero-VISA" strains (9), which represent the great majority of VISA isolates worldwide (5) and were proposed to be precursors of the less frequent homogeneously resistant VISA strains $(9,26)$. In cultures of hetero-VISA strains, the great majority of cells have low vancomycin MIC values, close to those of susceptible strains. However, the same cultures also contain low-frequency subpopulations of bacteria with increased vancomycin MIC value. Analysis of a collection of MRSA isolates recovered from hospitals in New York City identified numerous heterogeneously vancomycin-resistant strains. All of these isolates, including PC1 through PC3, belonged to the New York/Japan clone of MRSA (ST5, SCCmec II), which was widely spread in hospitals of New York, New Jersey, and Pennsylvania in the late 1990s (27).

Abnormal phenotypes of VISA isolates. All VISA isolates described above share some abnormal properties, including an excess of cell wall material (best visualized in electron microscopic thin sections), an aberrant separation of daughter cells at the end of cell division, and altered (most often decreased) rates of autolysis $(5,9-12,28,29)$.

Genetic/biochemical basis of VISA-type resistance. Elucidation of the genetic basis of VISA-type resistance requires the availability of both the resistant and the isogenic susceptible (parental) isolates recovered prior to the beginning of chemotherapy - ideally from the same patient. Comparison of such susceptible and resistant isolates can then be used to identify the altered genetic determinant in the VISA strain. This approach has identified a variable number and kinds of mutations in different VISA isolates (18, 28, 30-40).

The most frequently mutated determinants identified in 22 clinical VISA isolates from the collection of the CDC and the New York City Department of Health and Mental Hygiene were the walk $R$, vraSR, and $r p o B$ genes (31). In another study, the predominant genetic change was in the $y v q F / v r a S R$ system (41), while Watanabe et al. showed that most of the 38 VISA strains recovered from 10 different countries carried a mutated rpoB (42). In most, but not all, cases, the relevant mutated genes appear to be directly or indirectly involved with the biosynthesis/metabolism of the staphylococcal cell wall, specifically, two-component sensory regulatory systems controlling the transcription of genes in cell wall synthesis $(32,33,43)$. However, mutations in the RNA polymerase gene $r p o B$ were also detected in VISA isolates (42).

At least some of the extensive transcriptional changes observed in VISA strains could also be induced by treating the corresponding parental strain with vancomycin (44).

Identification of genetic stages in the development of VISA-type resistance by whole genome sequencing. In an attempt to better understand the evolution of VISA mutants, the emergence of VISA-type antibiotic resistance was followed in a patient undergoing extensive chemotherapy (36). Data available on this case suggest that the primary infection site was most likely the heart valve. Isolates were recovered from the blood stream of the patient at different times during chemotherapy and were tested for the MIC values of vancomycin. The MRSA strain causing the infection was a single locus variant of the multidrug-resistant New York/Japan clone (ST105), a frequent cause of hospital-acquired infections. The initial isolate (JH1) had a vancomycin MIC value of $1.0 \mu \mathrm{g} / \mathrm{ml}$. In the second isolate, $\mathrm{JH} 2$, the vancomycin MIC value increased to $4.0 \mu \mathrm{g} / \mathrm{ml}$. In the sixth isolate, JH6, the vancomycin MIC value reached $8 \mu \mathrm{g} / \mathrm{ml}$. Full genome sequencing was done on the last isolate, JH9 (recovered just before the patient expired), and on the initial fully susceptible isolate JH1, and the rate of appearance of the total of 33 mutated genes identified in JH9 was determined in the early and intermediate isolates by PCR sequencing (36). Consecutive isolates showed a gradual increase in vancomycin MIC value accompanied by a stepwise appearance and accumulation of 16 of the 33 mutations identified in strain JH9 (36). Determining which of these mutations actually contribute to the stepwise increase in vancomycin resistance will require complementation experiments. Also, the nature of the "excess" mutations in JH9, i.e., the 17 genetic alterations that appear only in isolate JH9, remains to be determined. These 17 mutations do not appear in isolate JH14, which had the same vancomycin MIC value as JH9 but was recovered directly from the infected heart valve of the patient during a heart valve replacement surgery performed late in the clinical history of this infection. The excess genetic alterations may represent mutations that contributed to the survival of the VISA strain JH9 in the hostile host environment.

In two recent studies similar in design to that used by Mwangi et al. (36), whole genome sequencing was done on each of the MRSA isolates recovered at various times from the blood stream of patients undergoing chemotherapy with various antimicrobial agents including vancomycin. In one of these studies, full genome sequencing was done on each of six consecutive isolates of the Brazilian clone of MRSA (ST239), which were recovered from the patient over a period of 77 days (45). In the second study, isolates were recovered and sequenced from a patient with a bloodstream infection by the Taiwan clone of MRSA (ST59) (30). These studies allowed identification of the emergence and shifts in the composition of entire subpopulations of bacteria in response to antibiotic treatment, including conversion of vancomycin-susceptible cells to hetero-VISA, followed by the eventual emergence of VISA mutants and the acquisition of multiple mutations in genes that control cell wall synthesis and autolysis $(30,45)$.

Another such genetic comparison of parental and corresponding VISA isolates was recently described in an MRSA strain belonging to the widely spread USA300 clone (ST8) (28). In this case, the parental, vancomycin-susceptible strain SG-S (recovered from the patient before the beginning of therapy), the VISA isolate SG-R, and a spontaneous revertant of the VISA strain (which had recovered the normal parental level of vancomycin susceptibility) were available for comparison. The SG-S and SG-R isolates showed differences in five genetic determinants, of which one, the mutated $y v q F$ (a regulator of the vraSR genetic system involved in the control of cell wall synthesis), was confirmed by complementation experiments to be responsible for the increased vancomycin MIC value. This was also consistent with the single genetic change identified in the revertant strain, which carried a complementary mutation in $\operatorname{vraSR}(28)$. The role of the four other mutations identified in SG-R is not known. 
Multiple mutations, in addition to the mutated gene responsible for the VISA phenotype, have been identified in several genetically characterized VISA isolates $(18,28,33,36)$. The contribution of these mutated genes to the VISA phenotype is not clear. In many cases, complementation experiments with the determinant responsible for the VISA phenotype have led to a complete recovery of the wild-type (parental) phenotype of the bacterium, but the extra mutations were still retained by the cells $(18,28,33,40,46)$. These extra mutations may represent alterations in genes that contribute to the survival and/or progress of the invading bacterium en route to anatomical sites of infection in the antibiotic treated host.

VISA mutants in epidemic MRSA clones. VISA-type mutants have now been identified in every one of the major epidemic clones of MRSA $(4,28,30,36,47-53)$. Cross-infection with VISA isolates has been described only in two reports, one from a French and another from an Italian hospital in which "epidemic" VISA strains appeared and spread in the hospital environment $(54,55)$. Clearly, acquisition of traits that would make VISA strains epidemic would be of concern.

The mutated genes identified in the VISA mutants of various MRSA clones varied considerably in nature. Therefore, it is conceivable that the particular mutated genes responsible for the VISA phenotype may be unique to the particular MRSA lineage, i.e., each MRSA clone may have a preferred genetic pathway to VISA-type resistance; however, an experimental test disproved this hypothesis. The MRSA strain JH1 (belonging to the New York/ Japan clonal lineage) was used to test whether vancomycin selection in the laboratory and in an experimental system mimicking in vivo exposure of bacteria to vancomycin would produce VISA mutants with genetic change in the same determinants that were identified in VISA-type mutants of strain JH1 that emerged in vivo in a patient during chemotherapy by vancomycin (36). While VISA mutants were recovered from strain JH1 in both the in vitro and semi-in vitro systems, the genetic alterations involved were different from the mutations identified in the in vivo VISA derivatives of strain JH1 (56). Similar conclusions were reached in several additional studies $(33,46)$.

VISA isolates were also identified in methicillin-susceptible S. aureus (MSSA) (57).

Decreased virulence and VISA-type resistance. Given the frequent therapeutic failure of VISA infections, the demonstration of decreased virulence in all VISA isolates (18-21) tested so far came as a surprise. In the VISA strain SG-R, belonging to the MRSA clone USA300, there was massive downregulation in the transcription of virulence determinants, including seven of the major virulence genes of $S$. aureus. Most importantly, normal or even above normal transcription of the same genes was obtained when the resistant isolate SG-R was "converted" to vancomycin susceptibility - by complementation experiments in the laboratory. These experiments demonstrate that decreased virulence was associated with the same mutated gene that was also responsible for the increased vancomycin MIC value. It was proposed that downregulation of virulence may represent a "stealth" strategy of the bacteria to evade surveillance by the host immune system (28).

Arrival of VRSA. The first $S$. aureus isolates exhibiting high-level vancomcyin resistance ( $\mathrm{MIC} \geq 16 \mu \mathrm{g} / \mathrm{ml}$ ) were described in 2002 in Michigan and Pennsylvania and in 2004 in New York (8). The strains carried plasmid-borne copies of the transposon Tn1546, which was acquired from vancomycin-resistant Enterococcus faecalis. Most strains appeared in a unique epidemiological scenario in diabetic wounds of patients that were infected by both vancomycin- resistant enterococci (VRE) and VRSA. As of the end of 2013 the number of VRSA isolates in the United States was 13 (58). Twelve of the thirteen Tn1546-containing S. aureus strains belong to the clonal complex CC5, an MRSA lineage that has been repeatedly isolated from clinical specimens (59). The most recently identified VRSA, which was recovered in Delaware, belongs to the lineage USA1100 (CC30) (58). Full genome sequencing of VRSA isolates has been described $(59,60)$. A VRSA isolate was also recently identified in Portugal, also in the diabetic wound of a patient (61). Additional reports from other parts of the world have not yet been confirmed.

The biochemical mechanism of action of vancomycin is based on the high affinity of this antibiotic for the D-alanyl-D-alanine (D-ala-D-ala) residue, a ubiquitous component of the bacterial cell wall precursor Lipid II. The mechanism of resistance identified in the Tn1546-based antibiotic resistance was shown to involve alteration of this dipeptide residue from $\mathrm{D}$-ala-D-ala to D-alanylD-lactate (D-ala-D-lac), a dipeptide with substantially lower affinity for the antibiotic $(62,63)$.

Biochemical studies with the MRSA strain COL carrying plasmid-borne copies of Tn1546 demonstrated that this strain produced the same altered cell wall already described in VRE strains. The terminal D-ala-D-ala residues in the resistant bacterium were replaced by D-ala-D-lac, a structure to which the antibiotic has a greatly decreased affinity (64). This particular strain also carried the mec $A$ determinant. Interestingly, the gene product of the mec $A$ determinant penicillin-binding protein 2A (PBP2A), a key component of the $\beta$-lactam resistance mechanism in MRSA, was unable to utilize the structurally altered cell wall precursor. Cell wall synthesis utilizing the altered D-ala-D-lac cell wall precursors was catalyzed in this strain by the native PBP2 of S. aureus (64). These findings indicate some degree of antagonism between the mecAdependent and Tn1546-based resistance mechanisms.

A model for common features of vancomycin resistance in VISA and VRSA. Since genetic and other evidence indicates that the VISAtype resistance also involves the staphylococcal cell wall, it was tempting to speculate about a biochemical mechanism in VISAtype resistance in terms of some modification of cell wall structure. In order to allow cell wall synthesis to continue in VISA strains in the presence of vancomycin, the VISA mutants must somehow protect Lipid II, which carries the building blocks of cell wall peptidoglycan terminating with the D-ala-D-ala residues. One way of achieving this could be to introduce into the mature cell wall of VISA isolates an excess of D-ala-D-ala-terminating cell wall chains, which could then act as false targets for the antibiotic and trap vancomycin at sites that are distant from the sites of bacterial cell wall biosynthesis. Mutations that decrease crosslinking of the staphylococcal cell wall could potentially produce excess D-alaD-ala residues. In fact, chemical analysis of the cell wall of strain JH9, one of the VISA mutants recovered from the blood stream of a patient who underwent extensive chemotherapy, was shown to contain peptidoglycan with decreased crosslinking and with greatly decreased activity of PBP4, a transpeptidase with an important role in the crosslinking of staphylococcal peptidoglycan (29). A gradual decrease in the crosslinking of peptidoglycan, i.e., the production of free D-ala-D-ala residues, was also demonstrated in laboratory mutants of the MRSA strain COL that were selected for increasing levels of vancomycin resistance (65). However, analysis of the cell wall composition of strain SG-R, another genetically characterized VISA isolate, did not show similar extensive alterations in the cell wall peptidoglycan (28). A selective enrichment of peptidoglycan 
in D-ala-D-ala residues in the proximity of cell wall synthetic sites may not be resolvable by currently used analytical techniques and is likely to require more complex experimental design (9).

In an experimental test of the "false target" hypothesis described above, fluorescence-labeled vancomycin was used to determine the amount of antibiotic bound by resistant and susceptible $S$. aureus isolates. Both laboratory mutants and VISA isolates bound more vancomycin; however, the amounts bound were not proportional to the MIC value of the bacteria (66). In a more sophisticated design of the vancomycin binding experiment, it was possible to demonstrate that in VISA strains, the rate of arrival of vancomycin molecules to sites of staphylococcal cell wall synthesis at the bacterial septum was delayed in resistant bacteria due to the presence of excess D-ala-Dala residues, which could capture and slow down the progress of the antibiotic to the site of cell wall biosynthesis. In this model, the false binding sites (D-ala-D-ala residues) that actually contribute to the increased MIC value are located close to the cell wall synthetic sites at the bacterial septum. With this modification of the model taken into account, the vancomycin binding test produced data that were consistent with the vancomycin MIC value of the bacteria (66).

\section{Summary}

The worldwide spread of multidrug-resistant MRSA clones during the past several decades has led to the frequent use of vancomycin to treat MRSA infections. This greatly increased selective pressure resulted in the emergence of MRSA isolates with reduced susceptibility to vancomycin - the so-called VISA strains - and, most recently, to the appearance of VRSA strains with high-level resistance to the antibiotic. VISA strains represent bacterial mutants with modestly increased vancomycin MIC $(3-8 \mu \mathrm{g} / \mathrm{ml})$, slightly greater than the susceptibility breakpoint. VISA strains emerge in vivo during the chemotherapy of patients with vancomycin. We describe the clinical history of a vancomycin-treated MRSA infection, which illustrates the stepwise appearance of mutants initially with a heterogeneous and subsequently with a more homogeneous VISA-type resistance. VISA isolates have been identified in each one of the major multidrug-resistant MRSA clones, and genetic analysis identified mutations in determinants that control the biosynthesis of bacterial cell wall and/or mutations in the ribosomal gene $r p o B$ as the most frequent genetic alterations associated with the VISA phenotype. The most recently identified VRSA isolates with a vancomycin MIC $\geq 100 \mu \mathrm{g} / \mathrm{ml}$ were identified in diabetic wounds coinfected by resistant $E$. faecalis and MRSA. In spite of their basic differences, the biochemical mechanisms of VISA and VRSA strains appear to share several common features.

\section{Acknowledgments}

This work was supported by a grant from the US Public Health Service (2 RO1 AI457838-14) awarded to A. Tomasz.

Address correspondence to: Alexander Tomasz, The Rockefeller University, Laboratory of Microbiology, 1230 York Avenue, New York, New York 10065, USA. Phone: 212.327.8278; Fax: 212.327.8688; E-mail: tomasz@mail.rockefeller.edu.
1. DeLeo FR, Chambers HF. Reemergence of antibiotic-resistant Staphylococcus aureus in the genomics era. J Clin Invest. 2009;119(9):2464-2474.

2. Maple PA, Hamilton-Miller JM, Brumfitt W. Worldwide antibiotic resistance in methicillin-resistant Staphylococcus aureus. Lancet. 1989;1(8637):537-540.

3. Levine DP. Vancomycin: a history. Clin Infect Dis. 2006;42(1 suppl 1):S5-S12.

4. Hiramatsu K, Hanaki H, Ino T, Yabuta K, Oguri T, Tenover FC. Methicillin-resistant Staphylococcus aureus clinical strain with reduced vancomycin susceptibility. J Antimicrob Chemother. 1997;40(1):135-136.

5. Howden BP, Davies JK, Johnson PD, Stinear TP, Grayson ML. Reduced vancomycin susceptibility in Staphylococcus aureus, including vancomycinintermediate and heterogeneous vancomycin-intermediate strains: resistance mechanisms, laboratory detection, and clinical implications. Clin Microbiol Rev. 2010;23(1):99-139.

6. Linares J. The VISA/GISA problem: therapeutic implications. Clin Microbiol Infect. 2001;7(suppl 4):8-15.

7. Fridkin SK, et al. Epidemiological and microbiological characterization of infections caused by Staphylococcus aureus with reduced susceptibility to vancomycin, United States, 1997-2001. Clin Infect Dis. 2003;36(4):429-439.

8. Sievert DM, Rudrik JT, Patel JB, McDonald LC, Wilkins MJ, Hageman JC. Vancomycin-resistant Staphylococcus aureus in the United States, 20022006. Clin Infect Dis. 2008;46(5):668-674.

9. Hiramatsu K. Vancomycin-resistant Staphylococcus aureus: a new model of antibiotic resistance. Lancet Infect Dis. 2001;1(3):147-155.

10. Howden BP, Peleg AY, Stinear TP. The evolution of vancomycin intermediate Staphylococcus aureus (VISA) and heterogeneous VISA. Infect Genet Evol. 2014;21:575-582.

11. Pfeltz RF, Wilkinson BJ. The escalating challenge of vancomycin resistance in Staphylococcus aureus. Curr Drug Targets Infect Disord. 2004;4(4):273-294.

12. Walsh TR, Howe RA. The prevalence and mechanisms of vancomycin resistance in Staphylococcus aureus. Annu Rev Microbiol. 2002;56:657-675.

13. Sieradzki K, Roberts RB, Haber SW, Tomasz A. The development of vancomycin resistance in a patient with methicillin-resistant Staphylococcus aureus infection. NEngl J Med. 1999;340(7):517-523.

14. Cameron DR, Howden BP, Peleg AY. The interface between antibiotic resistance and virulence in Staphylococcus aureus and its impact upon clinical outcomes. Clin Infect Dis. 2011;53(6):576-582.

15 . Horne KC, et al. Prospective comparison of the clinical impacts of heterogeneous vancomycin-intermediate methicillin-resistant Staphylococcus aureus (MRSA) and vancomycin-susceptible MRSA. Antimicrob Agents Chemother. 2009;53(8):3447-3452.

16. Lalueza A, Chaves F, San Juan R, Daskalaki M, Otero JR, Aguado JM. Is high vancomycin minimum inhibitory concentration a good marker to predict the outcome of methicillin-resistant Staphylococcus aureus bacteremia? J Infect Dis. 2010;15(201):311-312; author reply 312-313.

17. Price J, Atkinson S, Llewelyn M, Paul J. Paradoxical relationship between the clinical outcome of Staphylococcus aureus bacteremia and the minimum inhibitory concentration of vancomycin. Clin Infect Dis. 2009;48(7):997-998.

18. Cameron DR, et al. Serine/threonine phosphatase Stp1 contributes to reduced susceptibility to vancomycin and virulence in Staphylococcus aureus. J Infect Dis. 2012;205(11):1677-1687.

19. Gao W, et al. The RpoB H(4)(8)(1)Y rifampicin resistance mutation and an active stringent response reduce virulence and increase resistance to innate immune responses in Staphylococcus aureus. J Infect Dis. 2013;207(6):929-939.

20. Majcherczyk PA, Barblan JL, Moreillon P, Entenza JM. Development of glycopeptide-intermediate resistance by Staphylococcus aureus leads to attenuated infectivity in a rat model of endocarditis. Microb Pathog. 2008;45(5-6):408-414.

21. Peleg AY, et al. Reduced susceptibility to vancomycin influences pathogenicity in Staphylococcus aureus infection. J Infect Dis. 2009;199(4):532-536.
22. Holland TL, Fowler VG, Fowler VG Jr. Vancomycin minimum inhibitory concentration and outcome in patients with Staphylococcus aureus bacteremia: pearl or pellet? J Infect Dis. 2011;204(3):329-331.

23. Holmes NE, Johnson PD, Howden BP. Relationship between vancomycin-resistant Staphylococcus aureus, vancomycin-intermediate $S$. aureus, high vancomycin MIC, and outcome in serious S. aureus infections. J Clin Microbiol. 2012;50(8):2548-2552.

24. van Hal SJ, Fowler VG, Fowler VG Jr. Is it time to replace vancomycin in the treatment of methicillinresistant Staphylococcus aureus infections? Clin Infect Dis. 2011;56(12):1779-1788.

25. Tomasz A, Nachman S, Leaf H. Stable classes of phenotypic expression in methicillin-resistant clinical isolates of staphylococci. Antimicrob Agents Chemother. 1991;35(1):124-129.

26. Liu C, Chambers HF. Staphylococcus aureus with heterogeneous resistance to vancomycin: epidemiology, clinical significance, and critical assessment of diagnostic methods. Antimicrob Agents Chemother. 2003;47(10):3040-3045.

27. Roberts RB, et al. Distribution of methicillin-resistant Staphylococcus aureus clones among health care facilities in Connecticut, New Jersey, and Pennsylvania. Microb Drug Resist. 2000;6(3):245-251.

28. Gardete $S$, et al. Genetic pathway in acquisition and loss of vancomycin resistance in a methicillin resistant Staphylococcus aureus (MRSA) strain of clonal type USA300. PLoS Pathog. 2012;8(2):e1002505.

29. Sieradzki K, Tomasz A. Alterations of cell wall structure and metabolism accompany reduced susceptibility to vancomycin in an isogenic series of clinical isolates of Staphylococcus aureus. J Bacteriol. 2003;185(24):7103-7110.

30. Chen CJ, Lin MH, Shu JC, Lu JJ. Reduced susceptibility to vancomycin in isogenic Staphylococcus aureus strains of sequence type 59: tracking evolution and identifying mutations by whole-genome sequencing. J Antimicrob Chemother. 2013;69(2):349-354.

31. Hafer C, Lin Y, Kornblum J, Lowy FD, Uhlemann AC. Contribution of selected gene mutations to 
resistance in clinical isolates of vancomycin-intermediate Staphylococcus aureus. Antimicrob Agents Chemother. 2012;56(11):5845-5851.

32. Howden BP, et al. Evolution of multidrug resistance during Staphylococcus aureus infection involves mutation of the essential two component regulator WalKR. PLoS Pathog. 2011;7(11):e1002359.

33. Howden BP, Stinear TP, Allen DL, Johnson PD, Ward PB, Davies JK. Genomic analysis reveals a point mutation in the two-component sensor gene graS that leads to intermediate vancomycin resistance in clinical Staphylococcus aureus. Antimicrob Agents Chemother. 2008;52(10):3755-3762.

34. Matsuo M, Cui L, Kim J, Hiramatsu K. 2013. Comprehensive identification of mutations responsible for hVISA-to-VISA conversion in laboratory-generated VISA strains from hVISA clinical strain Mu3 Antimicrob Agents Chemother. 2013;57(12):5843-5853.

35. McEvoy CR, et al. Decreased vancomycin susceptibility in Staphylococcus aureus caused by IS256 tempering of WalKR expression. Antimicrob Agents Chemother. 2013;57(7):3240-3249.

36. Mwangi MM, et al. Tracking the in vivo evolution of multidrug resistance in Staphylococcus aureus by whole-genome sequencing. Proc Natl Acad Sci US A. 2007;104(22):9451-9456.

37. Shoji $\mathrm{M}$, et al. walK and clpP mutations confer reduced vancomycin susceptibility in Staphylococcus aureus. Antimicrob Agents Chemother. 2011; 55(8):3870-3881.

38. Sakoulas G, et al. Accessory gene regulator (agr) locus in geographically diverse Staphylococcus aureus isolates with reduced susceptibility to vancomycin. Antimicrob Agents Chemother. 2002; 46(5):1492-1502

39. Sakoulas G, et al. Reduced susceptibility of Staphylococcus aureus to vancomycin and platelet microbicidal protein correlates with defective autolysis and loss of accessory gene regulator (agr) function. Antimicrob Agents Chemother. 2005;49(7):2687-2692.

40. Cui L, Neoh HM, Shoji M, Hiramatsu K. Contribution of vraSR and graSR point mutations to vancomycin resistance in vancomycin-intermediate Staphylococcus aureus. Antimicrob Agents Chemoth er. 2009;53(3):1231-1234.

41. Kato Y, Suzuki T, Ida T, Maebashi K. Genetic changes associated with glycopeptide resistance in Staphylococcus aureus: predominance of amino acid substitutions in YvqF/VraSR.J Antimicrob Chemother. 2010;65(1):37-45.

42. Watanabe Y, Cui L, Katayama Y, Kozue K, Hiramatsu K. Impact of rpoB mutations on reduced vancomycin susceptibility in Staphylococcus aureus. J Clin Microbiol. 2011;49(7):2680-2684.

43. Kuroda M, Kuroda H, Oshima T, Takeuchi F, Mori
H, Hiramatsu K. Two-component system VraSR positively modulates the regulation of cell-wall biosynthesis pathway in Staphylococcus aureus. Mol Microbiol. 2003;49(3):807-821.

44. McAleese F, et al. Overexpression of genes of the cell wall stimulon in clinical isolates of Staphylococcus aureus exhibiting vancomycin-intermediate $S$. aureus-type resistance to vancomycin. J Bacteriol. 2006;188(3):1120-1133.

45. van Hal SJ, et al. In vivo evolution of antimicrobial resistance in a series of Staphylococcus aureus patient isolates: the entire picture or a cautionary tale? J Antimicrob Chemother. 2014;69(2):363-367.

46. Passalacqua KD, Satola SW, Crispell EK, Read TD. A mutation in the PP2C phosphatase gene in a Staphylococcus aureus USA300 clinical isolate with reduced susceptibility to vancomycin and daptomycin. Antimicrob Agents Chemother. 2012;56(10):5212-5223

47. Jansen A, Turck M, Szekat C, Nagel M, Clever I, Bierbaum $\mathrm{G}$. Role of insertion elements and yycFG in the development of decreased susceptibility to vancomycin in Staphylococcus aureus. Int J Med Microbiol. 2007;297(4):205-215.

48. Sieradzki K, Leski T, Dick J, Borio L, Tomasz A Evolution of a vancomycin-intermediate Staphylococcus aureus strain in vivo: multiple changes in the antibiotic resistance phenotypes of a single lineage of methicillin-resistant $\mathrm{S}$. aureus under the impact of antibiotics administered for chemotherapy. J Clin Microbiol. 2003;41(4):1687-1693.

49. Smith TL, et al. Emergence of vancomycin resistance in Staphylococcus aureus. GlycopeptideIntermediate Staphylococcus aureus Working Group. NEngl J Med. 1999;340(7):493-501.

50. Gardete S, Aires-De-Sousa M, Faustino A, Ludovice AM, de Lencastre H. Identification of the first vancomycin intermediate-resistant Staphylococcus aureus (VISA) isolate from a hospital in Portugal. Microb Drug Resist. 2008;14(1):1-6.

51. Graber CJ, Wong MK, Carleton HA, Perdreau-Remington F, Haller BL, Chambers HF. Intermediate vancomycin susceptibility in a community-associated MRSA clone. Emerg Infect Dis. 2007;13(3):491-493.

52. Hageman JC, et al. Occurrence of a USA300 vancomycin-intermediate Staphylococcus aureus. Diagn Microbiol Infect Dis. 2008;62(4):440-442.

53. Howden BP, Johnson PD, Ward PB, Stinear TP, Davies JK. Isolates with low-level vancomycin resistance associated with persistent methicillin-resistant Staphylococcus aureus bacteremia. Antimicrob Agents Chemother. 2006;50(9):3039-3047.

54. Cassone M, Campanile F, Pantosti A, Venditti M, Stefani S. Identification of a variant "Rome clone" of methicillin-resistant Staphylococcus aureus with decreased susceptibility to vancomycin, responsible for an outbreak in an intensive care unit. Microb Drug Resist. 2004;10(1):43-49.

55. de Lassence A, et al. Control and outcome of a large outbreak of colonization and infection with glycopeptide-intermediate Staphylococcus aureus in an intensive care unit. Clin Infect Dis. 2006;42(2):170-178.

56. Vidaillac C, et al. Alternative mutational pathways to intermediate resistance to vancomycin in methicillin-resistant Staphylococcus aureus. J Infect Dis. 2013;208(1):67-74.

57. Pillai SK, Wennersten C, Venkataraman L, Eliopoulos GM, Moellering RC, Karchmer AW. Development of reduced vancomycin susceptibility in methicillin-susceptible Staphylococcus aureus. Clin Infect Dis. 2009;49(8):1169-1174.

58. Limbago BM, Kallen AJ, Zhu W, Eggers P, McDougal LK, Albrecht VS. Report of the 13th Vancomycin-resistant Staphylococcus aureus from the United States. J Clin Microbiol. 2014;52(3):998-1002.

59. Kos VN, et al. Comparative genomics of vancomycin-resistant Staphylococcus aureus strains and their positions within the clade most commonly associated with Methicillin-resistant S. aureus hospital-acquired infection in the United States. MBio. 2012;3(3);e00112-12.

60. Kobayashi SD, Musser JM, DeLeo FR. Genomic analysis of the emergence of vancomycin-resistant Staphylococcus aureus. MBio. 2012;26;3(4):e00170-12.

61. Melo-Cristino J, Resina C, Manuel V, Lito L, Ramirez M. First case of infection with vancomycin-resistant Staphylococcus aureus in Europe. Lancet. 2013;382(9888):205.

62. Arthur M, Courvalin P. Genetics and mechanisms of glycopeptide resistance in enterococci. Antimicrob Agents Chemother. 1993;37(8):1563-1571.

63 . Handwerger S, Skoble J. Identification of chromosomal mobile element conferring high-level vancomycin resistance in Enterococcus faecium. Antimicrob Agents Chemother. 1995;39(11):2446-2453.

64. Severin A, Wu SW, Tabei K, Tomasz A. Penicillinbinding protein 2 is essential for expression of highlevel vancomycin resistance and cell wall synthesis in vancomycin-resistant Staphylococcus aureus carrying the enterococcal vanA gene complex. Antimicrob Agents Chemother. 2004;48(12):4566-4573.

65. Sieradzki K, Tomasz A. Gradual alterations in cell wall structure and metabolism in vancomycinresistant mutants of Staphylococcus aureus. J Bacteriol. 1999;181(24):7566-7570.

66. Pereira PM, Filipe SR, Tomasz A, Pinho MG. Fluorescence ratio imaging microscopy shows decreased access of vancomycin to cell wall synthetic sites in vancomycin-resistant Staphylococcus aureus. Antimicrob Agents Chemother. 2007;51(10):3627-3633. 